\title{
Smoothened antagonist GDC-0449 (Vismodegib) inhibits proliferation and triggers apoptosis in colon cancer cell lines
}

\author{
CHUANQING WU ${ }^{1}$, SHAOBO $\mathrm{HU}^{2}$, JI CHENG ${ }^{1}$, GUOBIN WANG $^{1}$ and KAIXIONG TAO ${ }^{1}$ \\ Departments of ${ }^{1}$ Gastrointestinal Surgery and ${ }^{2}$ Hepatobiliary Surgery, Union Hospital, Tongji Medical College, \\ Huazhong University of Science and Technology, Wuhan, Hubei 430022, P.R. China
}

Received February 16, 2016; Accepted February 3, 2017

DOI: $10.3892 /$ etm.2017.4282

\begin{abstract}
The sonic hedgehog (Shh) pathway has been proven to be involved in embryonic development and cancer growth. GDC-0449, an antagonist of the hedgehog signaling receptor Smoothened (Smo), was recently approved by the US Food and Drug Administration as a prescription for skin basal cell carcinoma. However, the efficacy of GDC-0449 in the treatment of colon cancer and other malignancies, such as basal cell carcinoma and pancreatic cancer, has remained to be proven. The present study assessed the effect of GDC-0449 on the colon cancer cell lines Caco-2 and Ht-29. A Cell Counting Kit- 8 assay was applied to assess the cell proliferation rate and apoptosis was tested by flow cytometry. Reverse-transcription quantitative PCR and western blot analysis were used for analyzing expression levels of target genes. Cell proliferation was inhibited, while apoptosis was increased by GDC-0449, whereas the expression of B-cell lymphoma 2 (Bcl-2), a downstream target of Shh signaling, was decreased. Consistent with the inhibition of Gli1 expression, the cancer stem cell markers CD44 and ALDH were decreased in the presence of GDC-0449. In conclusion, GDC-0449 was shown to inhibit the replication of colon cancer cells and trigger apoptosis through downregulating Bcl-2. This may also influence the stemness of cancer stem cells as indicated by the decreased stem cell surface markers.
\end{abstract}

\section{Introduction}

Colon cancer, one of the most significant malignancies worldwide, is the second most common cause of cancer-associated mortality in the USA (1-3). While it has been known for hundreds of years, surgical resection and chemotherapy still

Correspondence to: Dr Kaixiong Tao, Department of Gastrointestinal Surgery, Union Hospital, Tongji Medical College, Huazhong University of Science and Technology, 1277 Jiefang Avenue, Wuhan, Hubei 430022, P.R. China

E-mail: tao_kaixiong@163.com

Key words: sonic hedgehog, smoothened, GDC-0449, colon cancer, B-cell lymphoma 2 dominate current regimens and have provided only limited improvement of the 5-year survival rate (4-6). The current knowledge on the molecular mechanisms of the genesis and progression of colon cancer is limited and its expansion may provide approaches for developing targeted regimens. Identification of novel molecular pathways may overcome this current impasse (7).

The sonic hedgehog (Shh) signaling pathway has become a focus of cancer research in recent years. Shh signaling is a highly evolutionarily conserved pathway $(8,9)$. It is initiated by the binding of the exo-secretion ligand Shh to its trans-membrane receptor Patched (Ptch), thereby decreasing its inhibitory effect on the downstream receptor Smoothened (Smo). Smo then travels into the primary cilium of the cell and triggers an intracellular signaling cascade. This cascade leads to activation and nuclear translocation of Gli2, a Gli family transcription factor. In parallel with Gli2 translocation to the nucleus, the expression of downstream factors of Shh, such as Gli1, is upregulated. Gli1 is a Shh transcriptional factor and has a vital role in cell proliferation, regulation of survival and determination of cell fate by turning on specific gene expression. Blocking of any component in the pathway, particularly the inhibition of Smo and Gli1, may lead to cell death and embryonic lethality, revealing their major function in development (9-11).

The Shh signaling pathway is responsible for embryogenesis and the association between embryogenesis and tumorigenesis has inspired investigations on whether Shh is involved in tumor development. Numerous lines of evidence have shown aberrant activation of the Shh signaling pathway in cancer patients and drug intervention to block the transduction has resulted in tumor shrinking and cancer cell apoptosis in numerous types of solid tumor (12-14). In vivo and in vitro, pancreatic cancer cells have a lower proliferative and a higher apoptotic rate after intervention with an Shh antagonist $(15,16)$. Following suppression by a specific inhibitor, malignant hepatic neoplasms also display a reduction in the degree of malignancy, such as slower proliferation and higher vulnerability to chemotherapy $(17,18)$. Based on the discovery of the association of Shh with cancer, several studies have shown that Shh signaling has an important role in colon cancer. Yoshikawa et al (19) assessed the expression of the Shh signaling proteins Shh, Ptch and Smo by immunohistochemistry and compared the expression rate in seventeen hyperplastic polyps, 24 adenomas 
of the colon, 69 adenocarcinomas (31 well-differentiated and 38 moderately-differentiated) and 30 normal colon samples, revealing that almost all adenomas (22 out of 23;96\%), expressed Shh. Furthermore, 4 of 17 hyperplastic polyps (24\%), 7 of 31 well-differentiated adenocarcinomas (23\%), 13 of 38 moderately-differentiated adenocarcinomas (34\%) and none of the 30 normal samples expressed Shh. The expression rate of Ptch and Smo gradually increased in accordance with the degree of tumor progression (19). Mazumdar et al (20) showed the importance of targeting the Gli genes downstream of Smo for terminating Hh-dependent survival, suggesting that Gli may constitute a molecular switch that determines the balance between cell survival and cell death in human colon carcinoma (20). Therefore, the present study further assessed the association between Shh and colon cancer with the aim of identifying whether blockage of the Shh signaling pathway may represent a feasible approach for treating colon cancer. With this regard, Shh signaling inhibitors may benefit colon cancer patients and improve their prognosis.

Among all of the Shh-associated targeting agents, GDC-0449 (Vismodegib), a newly synthesized Smo antagonist, has shown excellent clinical efficacy in treating basal cell skin cancer and has been approved for clinical usage by the Food and Drug Administration of the USA (FDA) $(21,22)$. To the best of our knowledge, there is little experimental and clinical evidence on whether it is effective in colon cancer, suggesting its potential use as a colon cancer therapeutic. Therefore, the present study focused the association between Shh and colon cancer, and on the possible clinical prospect of GDC-0449.

\section{Materials and methods}

Antibodies and reagents. Antibodies against Gli1 (ab134906) and B-cell lymphoma 2 (Bcl-2; ab32124) were purchased from Abcam (Shanghai, China). Total proteins were extracted with cell lysis buffer (P0013; Beyotime Institute of Biotechnology, Haimen, China). Protein concentration was determined using an enhanced BCA protein assay kit (Applygen Technologies, Inc., Beijing, China). The corresponding secondary antibody is Anti-Rabbit IgG H\&L (Alexa Fluor 488; ab150077; Abcam). All proteins were detected using chemiluminescence (Thermo Fisher Scientific Inc. Waltham, MA, USA). GDC-0449 was purchased from Pfizer Inc. (New York, NY, USA).

Cell culture. The Caco-2 and Ht-29 colon cancer cell lines were obtained from the American Type Culture Collection (Manassas, VA, USA) and cultured in Dulbecco's modified Eagle's medium (high glucose) supplemented with $10 \%$ fetal bovine serum (FBS) and $1 \%$ antibiotic-antimycotic at $37^{\circ} \mathrm{C}$ in a humidified atmosphere of $95 \%$ air and $5 \% \mathrm{CO}_{2}$. Passaging was performed when cultured cells were in a stable state and at $70-80 \%$ confluence. Routine replacement of medium and cell cryopreservation were performed in a standard and sterile environment.

Cell counting kit (CCK)-8 assay. Cells in complete medium (10\% FBS) were seeded into a 96-well plate (density of $10^{5}$ cells $/ \mathrm{ml}$; $100 \mu \mathrm{l}$ per well) with 2 wells used per group. After pre-culture for $24 \mathrm{~h}$, untreated cells, $250 \mu \mathrm{l}$ dimethylsulfoxide
(DMSO) or GDC-0449 at a final concentration of 5-50 $\mu \mathrm{M}$ was added to each well, followed by incubation for 24 or 48 h. CCK-8 reagent was then added to each well, followed by incubation for $4 \mathrm{~h}$. The optical density at $450 \mathrm{~nm}$ of each well was then measured and used for calculating the proliferation rate. The test was performed in duplicate and repeated at least 3 times.

Apoptosis assay. Cells were incubated with complete medium $(10 \% \mathrm{FBS})$ at a density of $10^{5}$ cells $/ \mathrm{ml}$. After pre-culture for $24 \mathrm{~h}$, untreated cells, $250 \mu \mathrm{l}$ DMSO or GDC-0449 at a final concentration of $5-50 \mu \mathrm{M}$ was added to each well, followed by incubation for 24 or $48 \mathrm{~h}$. Cells were trypsinized and collected by centrifugation at $300 \times \mathrm{g}$ for $5 \mathrm{~min}$ at $4^{\circ} \mathrm{C}$. Subsequently, cells were incubated with annexinV-FITC/propidium iodide (PI) for $15 \mathrm{~min}$ at room temperature after washing with $1 \mathrm{X}$ PBS. The percentage of cells in quadrant 4 (Q4; annexin V-FITC-positive and PI-negative) was assessed by flow cytometry (FACS Calibur; BD Biosciences, San Jose, CA, USA). The assay was repeated 3 times.

Western blot analysis. A standard western blot protocol was used. Protein extraction and bicinchoninic acid examination of the protein concentration were performed with domestic reagents. SDS-PAGE and subsequent immunologic reaction were routinely performed with appropriate reagents. All experiments were performed in triplicate (23).

Reverse-transcription quantitative polymerase chain reaction $(R T-q P C R)$ assay. Total RNA was extracted from colon cancer cells using TRIzol reagent and reverse transcription was performed using PrimeScript RT Master Mix (Takara, Dalian, China) according to the manufacturer's protocol. Complementary DNA was amplified with SYBR Premix Ex Taq (Takara). Primers specific for each gene were designed using Primer-BLAST (https://www.ncbi.nlm.nih. gov/tools/primer-blast/) and used to generate the PCR products. For the quantification of amplified genes, real-time qPCR was performed using an Applied Biosystems StepOne Plus Real-Time PCR System. The following gene-specific primers were used: Gli1 forward, 5'-TTC CTA CCA GAG TCC CAA GT-3' and reverse, 5'-CCC TAT GTG AAG CCC TAT TT-3'; CD44 forward, 5'-GTA GTA CAA CGG AAG AAA CA-3' and reverse, 5'-TGT GAG ATT GGG TTG AAG AA-3'; Bcl-2 forward, 5'-GAC TTC GCC GAG ATG TCC AG-3' and reverse, 5'-GGT GCC GGT TCA GGT ACT CA-3'; aldehyde dehydrogenase (ALDH) forward, 5'-TCC TCG GCT ACA TCA ACA CG-3' and reverse, 5'-CAT GCC ATC CTG CAC ATC TC-3'; and $\beta$-actin forward, 5'-TGG AAT CCT GTG GCA TCC ATG AAA C-3' and reverse, 5'-TAA AAC GCA GCT CAG TAA CAG TCC G-3'. Target sequences were amplified using the following thermocycling protocol: $95^{\circ} \mathrm{C}$ for $10 \mathrm{~min}$, followed by 40 cycles of $95^{\circ} \mathrm{C}$ for $15 \mathrm{sec}$ and $60^{\circ} \mathrm{C}$ for $1 \mathrm{~min}$. $\beta$-actin was used as an endogenous normalization control. All assays were performed in triplicate and relative expression levels were calculated on the basis of the $2^{-\Delta \Delta \mathrm{Cq}}$ method (24).

Statistical analysis. Values are expressed as the mean \pm standard deviation for each group. Differences between groups were analyzed by one or two-way analysis of variance using 


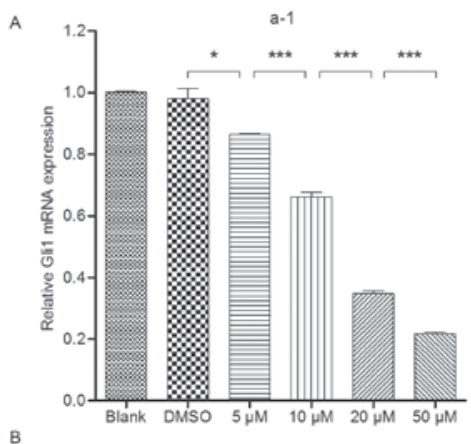

b-1

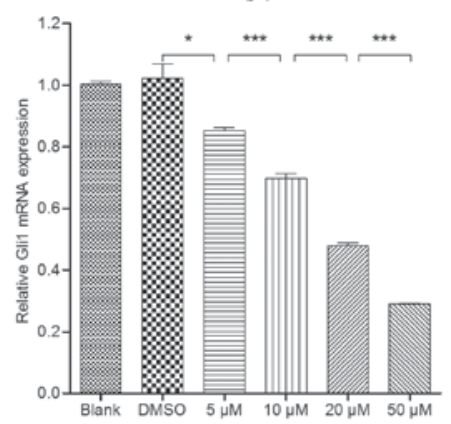

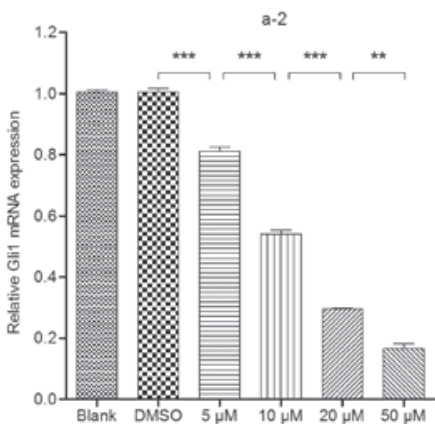

b-2

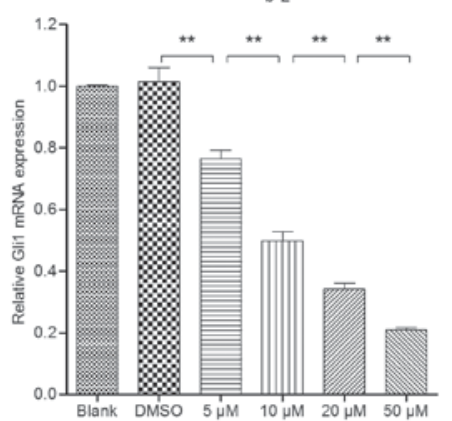

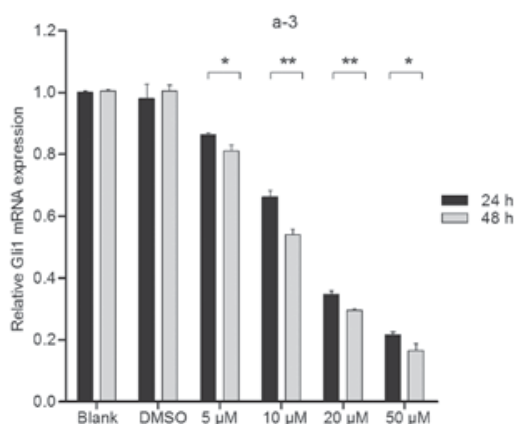

b-3

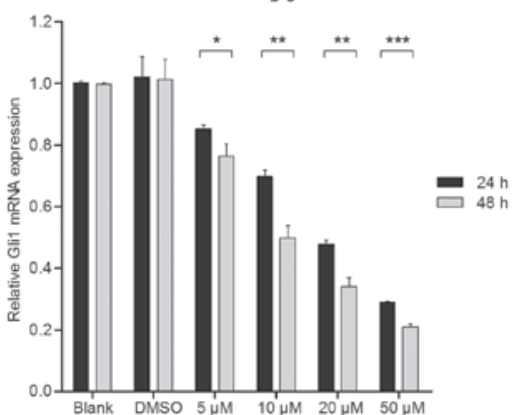

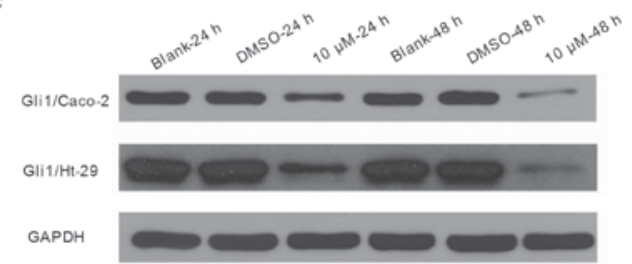

Figure 1. GDC-0449 decreases Gli1 expression in the Caco-2 and Ht-29 cell lines. Gli1 mRNA expression was detected by reverse-transcription quantitative polymerase chain reaction analysis. (A) Caco-2 cell line. Incubation of the blank, DMSO or 5-50 $\mu$ M GDC-0449 groups for (a-1) $24 \mathrm{~h}$ and (a-2) $48 \mathrm{~h}$. (a-3) Comparison of Glil mRNA expression in the Caco-2 cell line between 24 and $48 \mathrm{~h}$ at various GDC-0449 doses. (B) Ht-29 cell line. Incubation of the blank, DMSO or 5-50 $\mu$ M GDC-0449 groups for (b-1) 24 h and (b-2) 48 h. (b-3) Comparison of Gli1 mRNA expression in the Ht-29 cell line between 24 and $48 \mathrm{~h}$ at various GDC-0449 doses. Values are expressed as the mean \pm standard deviation. ${ }^{*} \mathrm{P}<0.05 ;{ }^{* * *} \mathrm{P}<0.01 ;{ }^{* * * *} \mathrm{P}<0.001$. (C) Western blot analysis of Gli1 protein in Caco-2 and Ht-29 cell lines. In the presence of $10 \mu \mathrm{M}$ GDC-0449, Gli1 protein expression was obviously decreased compared with the control group, with the effect at $48 \mathrm{~h}$ being greater than that at $24 \mathrm{~h}$. Expression levels in the two cell lines were similar. Results are representatives of three independent experiments. DMSO, dimethylsulfoxide.

GraphPad Prism statistical analysis software (GraphPad Software Inc., La Jolla, CA, USA). P<0.05 was considered to indicate a statistically significant difference.

\section{Results}

GDC-0449 suppresses Shh signaling in Caco-2 and Ht-29 cells through smo. GDC-0449 is a Smo antagonist approved by the FDA for treating basal cell skin carcinoma. Although it strongly inhibits Shh signaling in various types of cancer cell, its effect on colon cancer cells has remained to be fully clarified. In the present study, RT-qPCR analysis showed the expected effects of GDC-0449 in the colon cancer cell lines Caco-2 (Fig. 1A) and Ht-29 (Fig. 1B), as Gli1 expression was significantly decreased in a dose dependent manner $(5-50 \mu \mathrm{M})$. The strongest inhibition of Gli1 expression was at least $70 \%$ at $50 \mu \mathrm{M}$ and a significant difference was found between 24- and 48-h treatments for each dose $(\mathrm{P}<0.05)$. In line with these results, western blot analysis revealed that $10 \mu \mathrm{M}$ GDC-0449 was more efficient at $48 \mathrm{~h}$ than at $24 \mathrm{~h}$ (Fig. 1C).
GDC-0449 reduces the proliferation in human colon cancer cells. The effects of GDC-0499 on the proliferation of human colon cancer cell lines Caco-2 and Ht-29 was then assessed using a CCK-8 assay (Fig. 2). For each cell line, a significant decrease in the cell proliferation rate was revealed for the 24and 48-h drug treatments, which was dose-dependent. Further data analysis showed a significantly higher inhibition at $48 \mathrm{~h}$ compared with $24 \mathrm{~h}$. The proliferation was decreased by $35 \%$ of that in the control group.

GDC-0449 induces apoptosis in human colon cancer cells. Fluorescence-assisted cell sorting is widely used for cell apoptosis assays and the cell population in Q4 resembles the early apoptotic rate of cells. The present study examined whether GDC-0449 reduced the growth of colon cancer cells via inducing apoptosis. The expected increase in apoptosis after GDC-0449 treatment was found to be dose- and time-dependent (Fig. 3). The results may indicate that the inhibitory effect of GDC-0449 on human colon cancer cells is associated with its ability to induce apoptosis. 

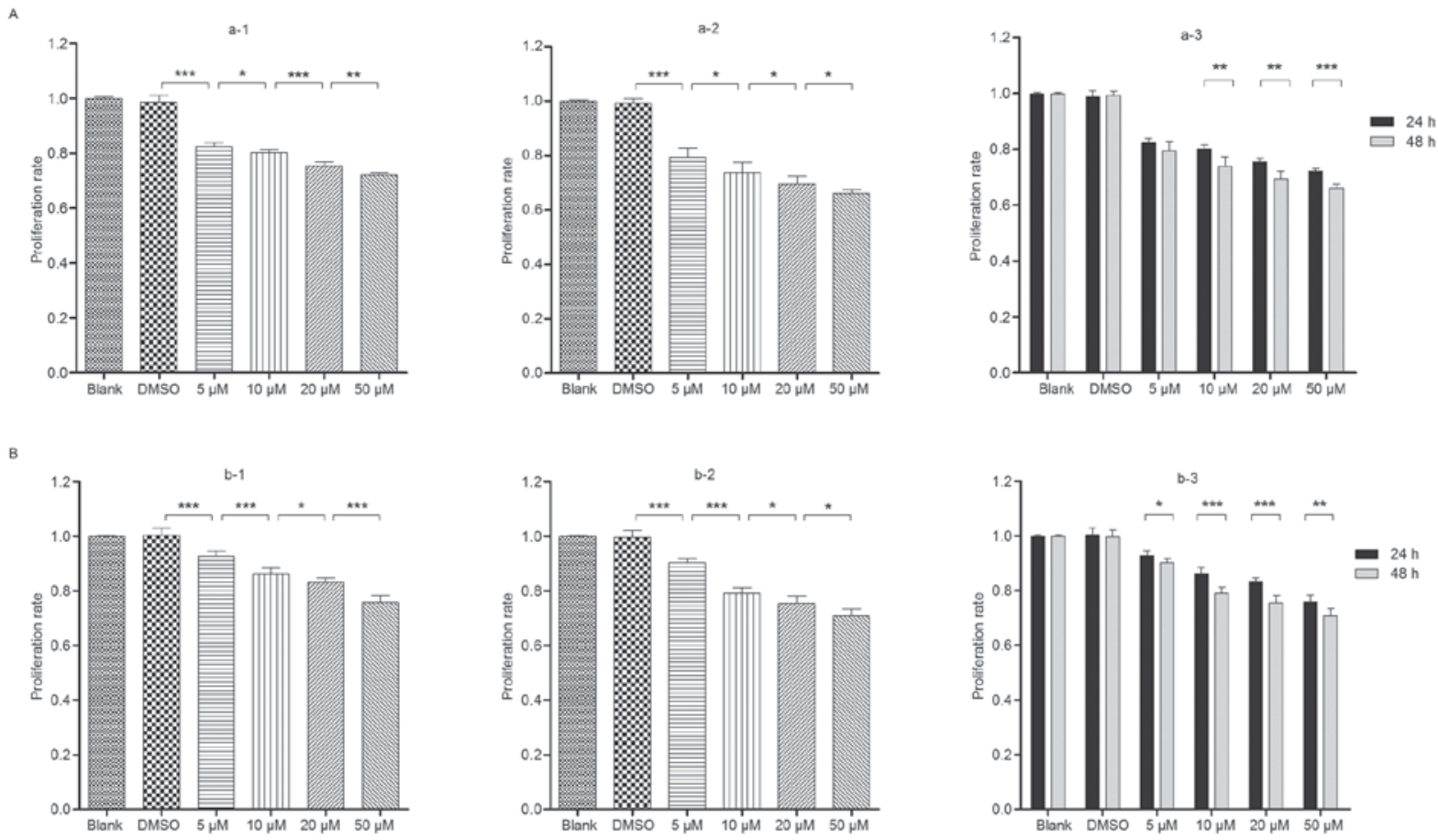

Figure 2. Effects of GDC-0449 on the proliferation rate of Caco-2 and Ht-29 cells. (A) Caco-2 cell line. Incubation of the blank, DMSO or 5-50 $\mu$ M GDC-0449 groups for (a-1) $24 \mathrm{~h}$ and (a-2) $48 \mathrm{~h}$. (a-3) Comparison of the proliferation rate of Caco-2 cells between 24 and $48 \mathrm{~h}$ at various GDC-0449 doses. (B) Ht-29 cell line. Incubation of the blank, DMSO or 5-50 $\mu$ M GDC-0449 groups for (b-1) $24 \mathrm{~h}$ and (b-2) 48 h. (b-3) Comparison of the proliferation rate of Ht-29 cells between 24 and $48 \mathrm{~h}$ at various GDC-0449 doses. Results were obtained by a Cell Counting Kit- 8 assay and were determined from three independent experiments. Values are expressed as the mean \pm standard deviation. ${ }^{*} \mathrm{P}<0.05 ;{ }^{* * *} \mathrm{P}<0.01 ;{ }^{* * * *} \mathrm{P}<0.001$. DMSO, dimethylsulfoxide.

GDC-0449 induces apoptosis via decreasing Bcl-2. The anti-apoptotic gene Bcl-2 is well known for its critical role in regulating cell cycle and death (25). However, to date, no substantial evidence has proven that GDC-0449 exerts its pro-apoptotic effect through the Bcl-2 pathway. The RT-qPCR results revealed a dose- and time-dependent decrease in Bcl-2 mRNA levels by GDC-0449 treatment in the two cell lines (Fig. 4A and B). The highest dose suppressed Bcl-2 expression by up to $60 \%$ of that in the control group. Western blot analysis verified that also the protein expression of Bcl-2 was decreased by GDC-0449 in a time-dependent manner for each cell line (Fig. 4C).

GDC-0449 decreases the expression of cancer stem cell (CSC) surface markers in colon cancer cells. CD44 and ALDH are recognized markers for colon CSCs (26). Caco-2 and $\mathrm{Ht}-29$ show different expression patterns of these markers (Fig. 5). RT-qPCR analysis demonstrated that CD44 and ALDH expression was similarly decreased by GDC-0449 in a dose- and time-dependent manner, comparable to other indicators mentioned above. The expression of the markers was decreased by $>70 \%$, suggesting GDC-0449 has a great anti-stem cell potential.

\section{Discussion}

The Shh signaling pathway is a hotspot of research on tumorigenesis and cancer metastasis (10). Initially, the involvement of Shh signaling in embryonic development of mammal species was discovered. Deprivation of certain molecules of this pathway was found to cause cerebellum atrophy and limb defects in rats (27-30). Since neoplasms and embryonic development share similar molecular signaling processes, numerous studies have examined and demonstrated the core role of Shh signaling in maintaining tumor growth and cancer cell survival (12). Vigorous proliferation was observed when the Shh pathway was upregulated in cancer cells, while inhibition of Shh had a suppressing effect (15). All of these phenomena highlight the clinical value of Shh-targeted therapy and the findings of the present study substantiated the theoretical basis for its future application in patients.

GDC-0449 (Vismodegib), a novel Smo inhibitor, has already been approved as a clinical prescription for basal cell skin carcinoma by the FDA, and it additionally displays a great inhibitory effect on numerous other types of solid tumor, including pancreatic cancer and malignancies of the central nervous system $(31,32)$. However, its effects on colon cancer have remained elusive. To the best of our knowledge, the present study was the first to prove that GDC-0449 exerts an effect on colon cancer cells, revealing a marked decrease in cell growth. Furthermore, different from the existing consensus that GDC-0449 mainly kills tumors by suppressing proliferation, the present study revealed that the pro-apoptotic effect of GDC-0449 had a leading role in its activity against the colon cancer cell lines. With the intervention by GDC-0449, the anti-apoptotic gene Bcl-2 was downregulated at the mRNA and protein level, which was in parallel with an increase in cell apoptosis observed by flow cytometry (33). 

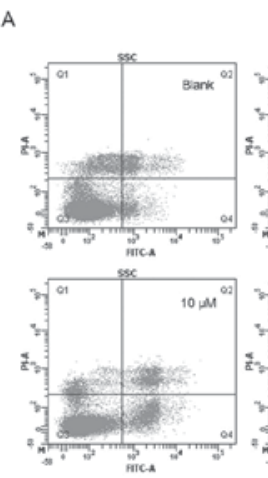

B

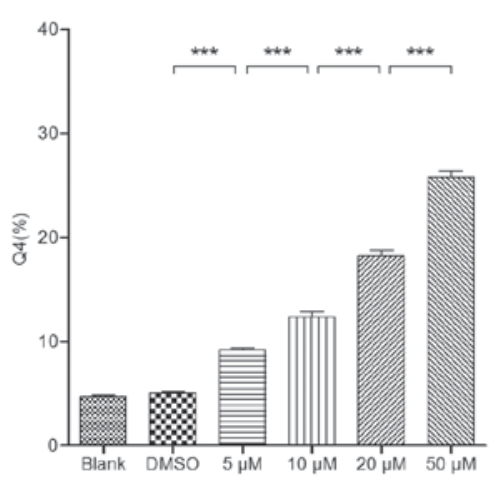

a-1
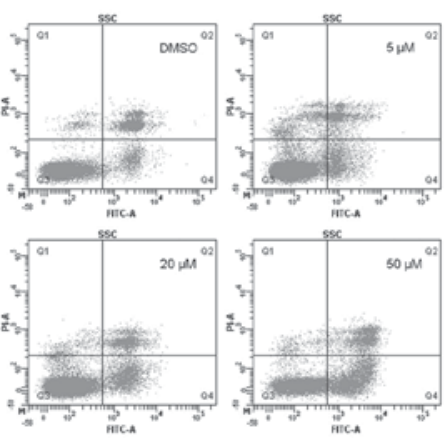
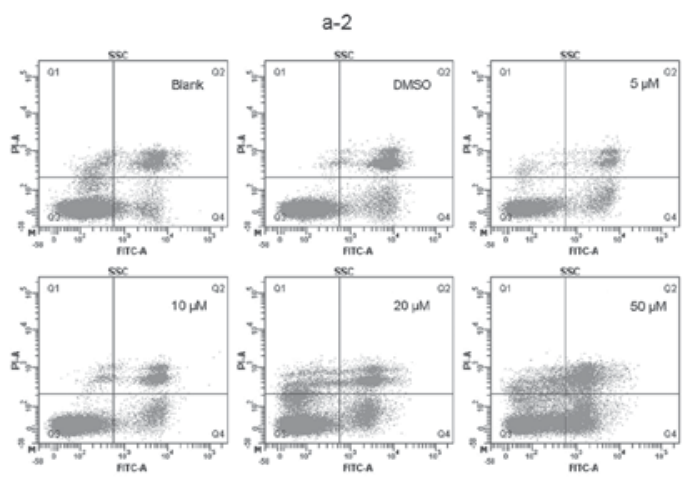

b-2

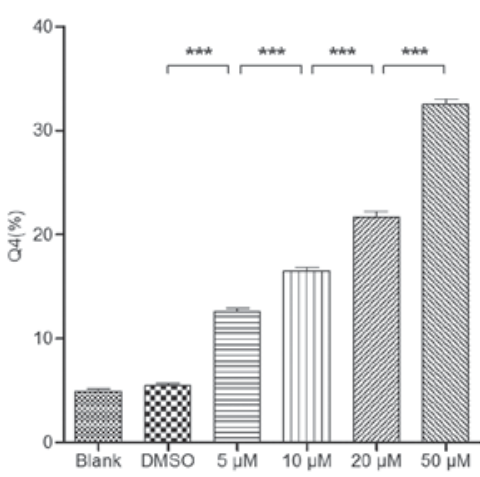

b-3

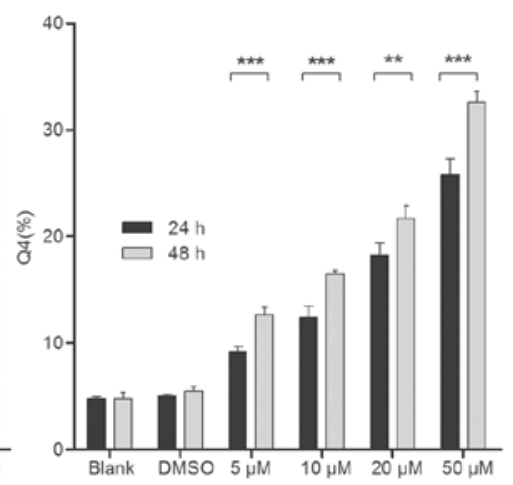

C c-1
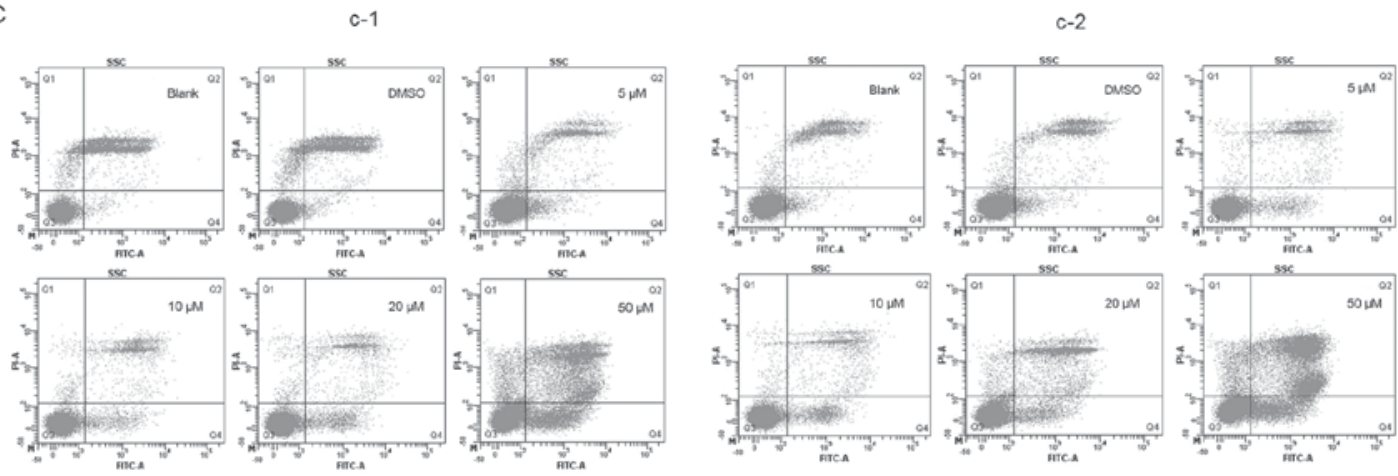

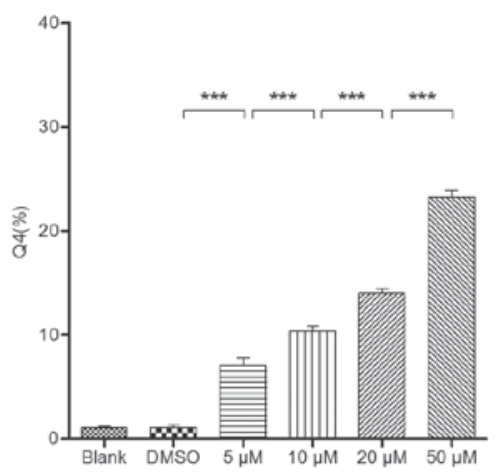

d-2

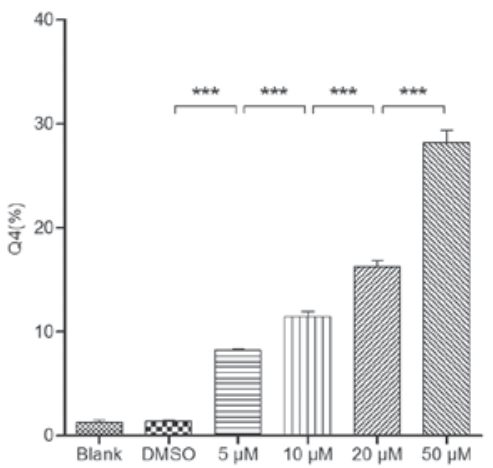

d-3

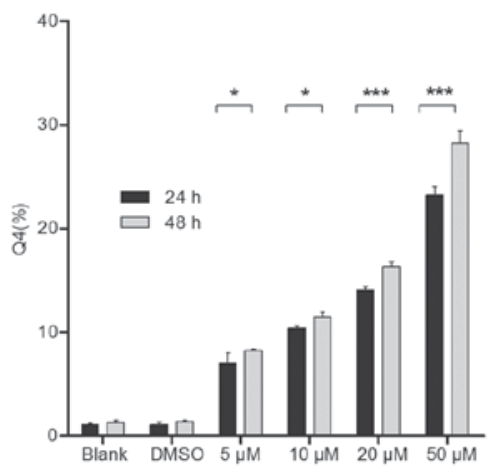

Figure 3. Effect of GDC-0449 on the apoptotic rate of Caco-2 and Ht-29 cells. (A) Flow cytometric dot plots for the Caco-2 cell line. Cells in Q4 represent early apoptotic cells. Incubation of the blank, DMSO or 5-50 $\mu$ M GDC-0449 groups for (a-1) 24 h and (a-2) 48 h. (B) Statistic diagrams showing the Q4 percentage in the Caco-2 cell line incubated for (b-1) $24 \mathrm{~h}$ and (b-2) $48 \mathrm{~h}$. (b-3) Comparison of the Q4 percentage in the Caco-2 cell line between 24 and $48 \mathrm{~h}$ with GDC-0449 treatment at various doses. (C) Flow cytometry dot plots for the Ht-29 cell line. Incubation of the blank, DMSO and 5-50 $\mu$ M groups for (c-1) $24 \mathrm{~h}$ and (c-2) $48 \mathrm{~h}$. (D) Statistic diagrams showing the Q4 percentage in the Ht-29 cell line incubated for (d-1) $24 \mathrm{~h}$ and (d-2) $48 \mathrm{~h}$. (d-3) Comparison of the Q4 percentage in the Ht-29 cell line between 24 and $48 \mathrm{~h}$ with GDC-0449 treatment at various doses. Results were determined from three independent experiments. Values are expressed as the mean \pm standard deviation. ${ }^{*} \mathrm{P}<0.05 ;{ }^{* *} \mathrm{P}<0.01 ;{ }^{* * *} \mathrm{P}<0.001$. DMSO, dimethylsulfoxide; PI, propidium iodide; FITC, fluorescein isothiocyanate; Q, quadrant. 

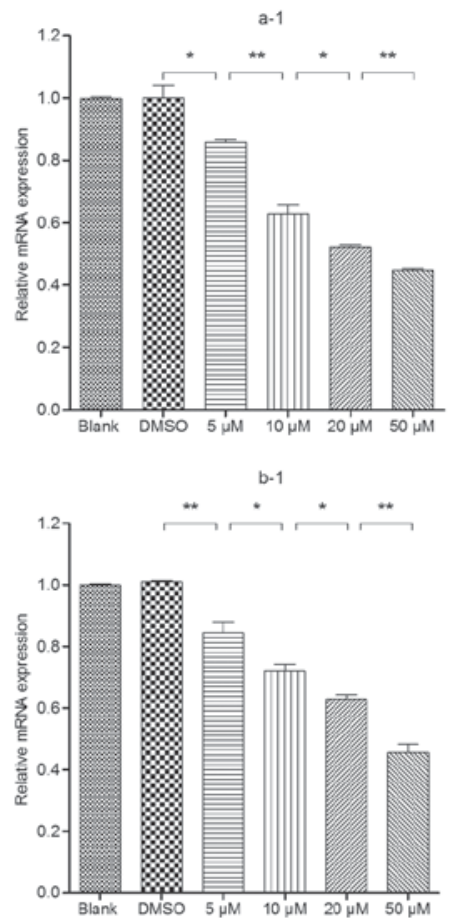
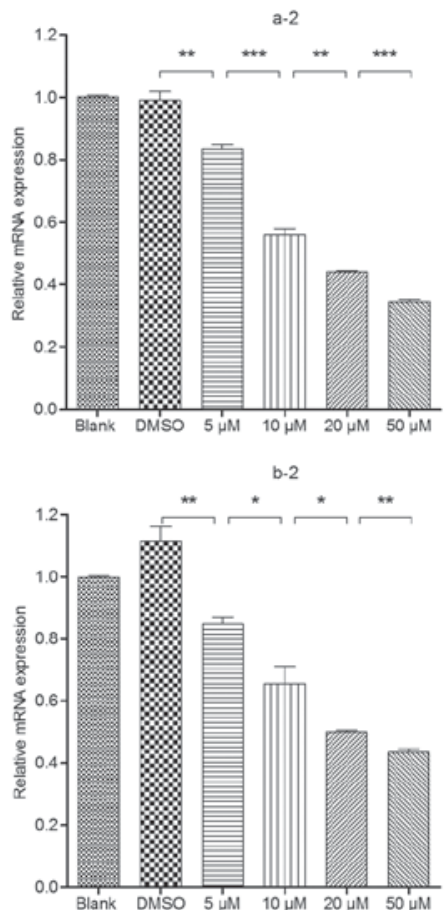

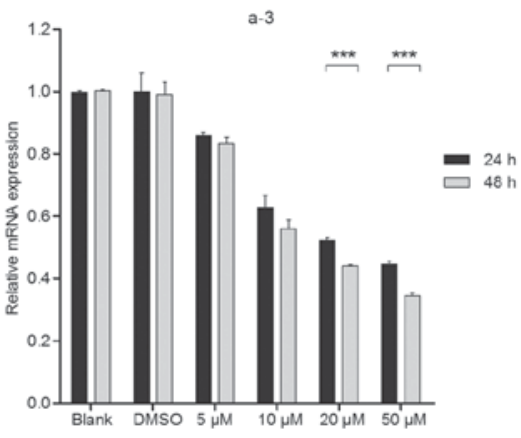

b-3

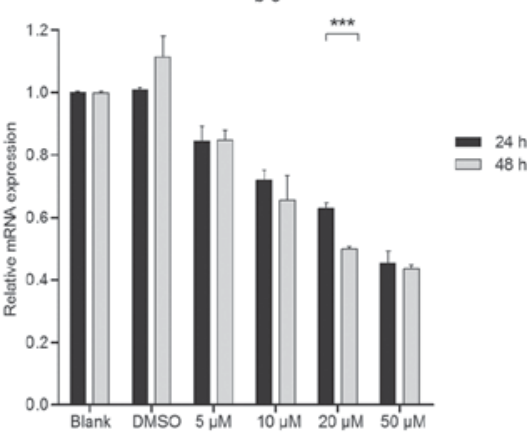

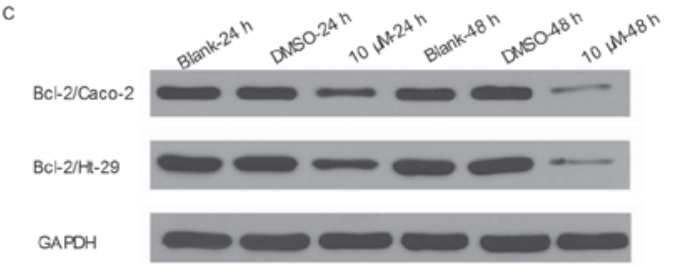

Figure 4. GDC-0449 decreases the expression of Bcl-2 in Caco-2 and Ht-29 cell lines. Reverse-transcription quantitative polymerase chain reaction analysis was used to detect Bcl-2 mRNA. (A) Caco-2 cell line. Blank, DMSO or 5-50 $\mu$ M GDC-0449 groups incubated for (a-1) 24 h and (a-2) 48 h. (a-3) Comparison of the expression of Gli1 mRNA in the Caco-2 cell line between 24 and $48 \mathrm{~h}$ for various GDC-0449 concentrations. (B) Ht-29 cell line. Blank, DMSO or 5-50 $\mu \mathrm{M}$ GDC-0449 groups incubated for (b-1) $24 \mathrm{~h}$ and (b-2) $48 \mathrm{~h}$. (b-3) Comparison of the expression of Gli1 mRNA in the Ht-29 cell line between 24 and $48 \mathrm{~h}$ for various GDC-0449 concentrations. (C) Western blot analysis of Bcl-2 protein in Caco-2 and Ht-29 cell lines. In the presence of $10 \mu \mathrm{M}$ GDC-0449, Bcl-2 protein expression was obviously decreased compared with the control group, with the effect at $48 \mathrm{~h}$ being greater than that at $24 \mathrm{~h}$. The two cell lines showed similar results. Results were determined from three independent experiments. Values are expressed as the mean \pm standard deviation. ${ }^{*} \mathrm{P}<0.05 ;{ }^{* * *} \mathrm{P}<0.01 ;{ }^{* * * *} \mathrm{P}<0.001$. DMSO, dimethylsulfoxide; Bcl-2, B-cell lymphoma 2.

Therefore, signaling via the 'Shh-Smo-Gli1-Bcl-2' cascade may represent the molecular pathway underlying the pharmacological effect of GDC-0449 on cancer cells. The present study may pave the road for the use of Shh-targeted regimens in colon cancer patients, providing additional options for physicians to improve their prognosis and possibly curing cancer in the future.

While the in vitro results of the present study are promising, the efficacy of GDC-0449 in colon cancer remains to be verified in vivo. Future in vivo and clinical studies may further support the use of GDC-0449 in colon cancer to enhance current treatments.

Previous studies suggested additional effects of Shh signaling on metastasis and CSCs besides its function in promoting cancer cell proliferation (34-36). Upregulated Shh signaling was found to facilitate metastasis and to be associated with poor prognosis in various types of cancer, possibly due to its role in regulating stemness of CSCs, which remains to be fully elucidated (37). Moreover, the present study found that GDC-0449 markedly decreased the expression of the stem cell surface markers CD44 and ALDH in colon cancer cell lines, suggesting the possible effect of Shh signaling on regulating the stemness of stem cells. Subsequent studies by our group will focus on in vivo proof and the role of Shh in CSCs.

In conclusion, the present study reported that GDC-0449, a Smo antagonist approved for treating basal cell skin carcinomas, is also potent in colon cancer cell lines. After drug treatment for 24 and $48 \mathrm{~h}$, Shh signaling was markedly inhibited with an obvious downregulation of Gli1. GDC-0449 was able to successfully trigger colon cancer cell apoptosis and thereby inhibit cell replication in a dose- and timedependent manner. Bcl-2, an anti-apoptotic gene, was further verified to be involved in the downstream molecular mechanism. The CSC surface markers CD44 and ALDH also displayed an evident decrease after GDC-0449 treatment, revealing its potential effects on CSCs. In conclusion, GDC-0449 showed marked anti-tumor efficacy in colon cancer cells in vitro by triggering cell apoptosis and inhibiting cell replication. 
A
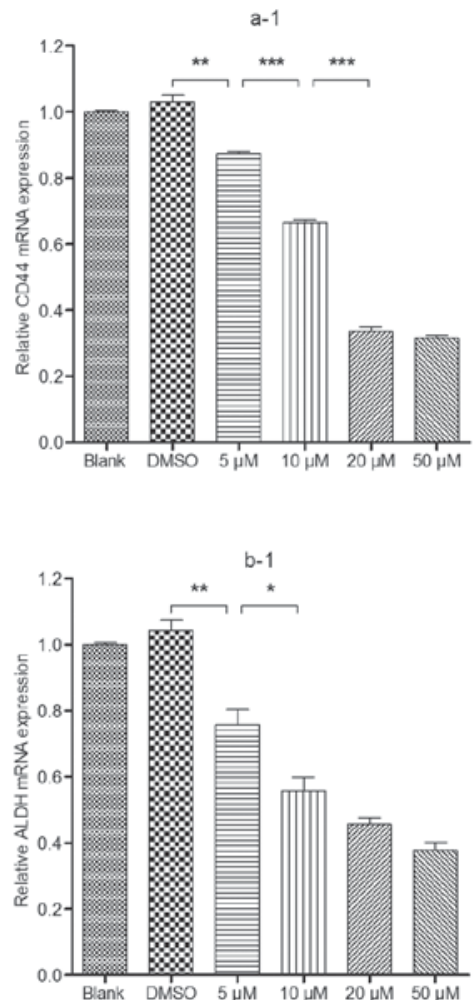

c

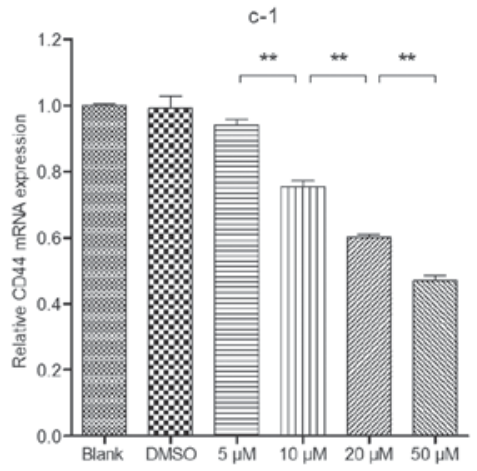

D

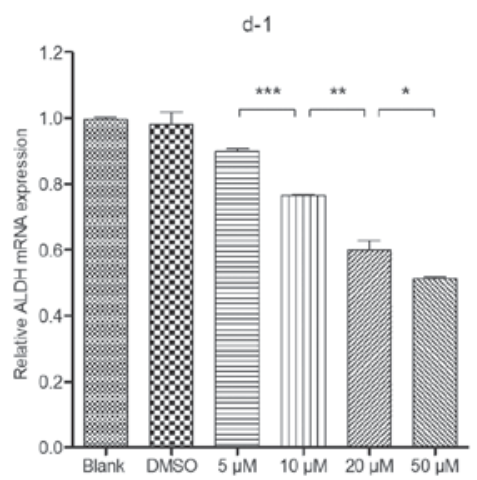

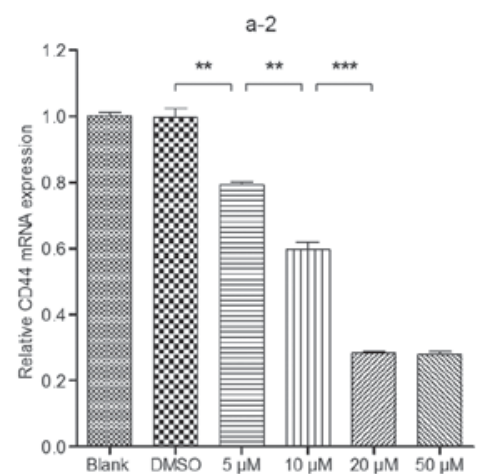
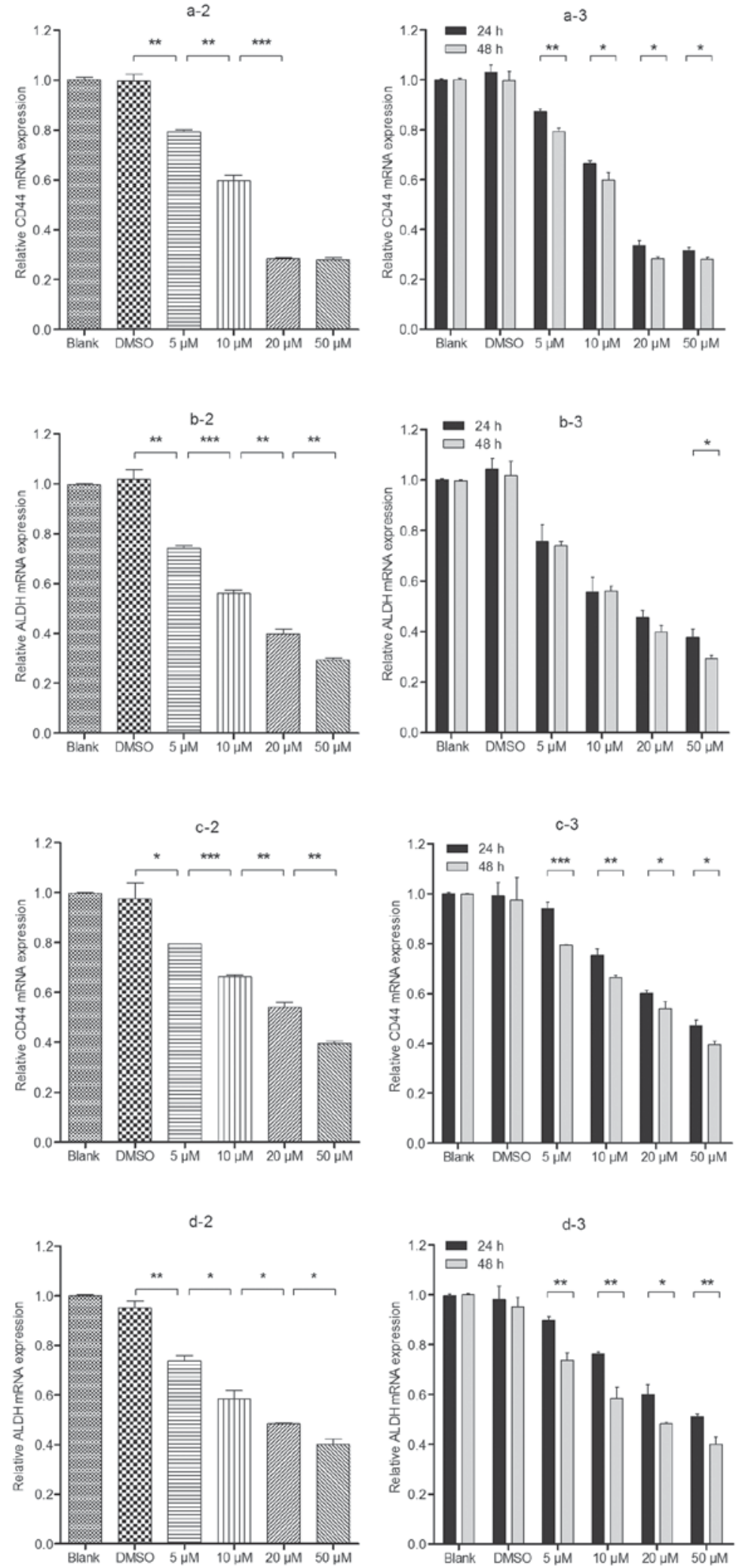

Figure 5. GDC-0449 causes decreases in CD44 and ALDH in the Caco-2 and Ht-29 cell lines. (A) Relative mRNA expression of CD44 in the Caco-2 cell line. Blank, DMSO or 5-50 $\mu$ M GDC-0449 groups incubated for (a-1) $24 \mathrm{~h}$ and (a-2) $48 \mathrm{~h}$. (a-3) Comparison of the expression of CD44 mRNA between 24 and $48 \mathrm{~h}$ of incubation with GDC-0449 at various doses. (B) Relative mRNA expression of ALDH in the Caco-2 cell line. Blank, DMSO or 5-50 $\mu$ M GDC-0449 groups incubated for (b-1) $24 \mathrm{~h}$ and (b-2) $48 \mathrm{~h}$. (b-3) Comparison of the expression of ALDH mRNA between 24 and $48 \mathrm{~h}$ of incubation with GDC-0449 at various doses. (C) Relative mRNA expression of CD44 in the Ht-29 cell line. Blank, DMSO or 5-50 $\mu \mathrm{M}$ GDC-0449 groups incubated for (c-1) $24 \mathrm{~h}$ and (c-2) $48 \mathrm{~h}$. (c-3) Comparison of the expression of CD44 mRNA between 24 and $48 \mathrm{~h}$ of incubation with GDC-0449 at various doses. (D) Relative mRNA expression of ALDH in the Ht-29 cell line. Blank, DMSO or 5-50 $\mu \mathrm{M}$ GDC-0449 groups incubated for (d-1) $24 \mathrm{~h}$ and (d-2) $48 \mathrm{~h}$. (d-3) Comparison of the expression of ALDH mRNA between 24 and $48 \mathrm{~h}$ of incubation with GDC-0449 at various doses. Results were determined from three independent experiments. Values are expressed as the mean \pm standard deviation. ${ }^{*} \mathrm{P}<0.05 ;{ }^{* *} \mathrm{P}<0.01 ;{ }^{* * *} \mathrm{P}<0.001$. DMSO, dimethylsulfoxide; ALDH, aldehyde dehydrogenase. 


\section{Acknowledgements}

This work was supported by the Young Teacher Foundation of Independent Innovation Fund of Huazhong University of Science and Technology (grant no. 2015QN197 to Chuanqing $\mathrm{Wu})$ and the National Natural Science Foundation of China (grant nos. 81600401 to Chuanqing $\mathrm{Wu}$ and 81172294 to KaiXiong Tao).

\section{References}

1. Zhu C, Bassig BA, Zaridze D, Boyle P, Dai M, Li Q and Zheng T: A birth cohort analysis of the incidence of ascending and descending colon cancer in the United States, 1973-2008. Cancer Causes Control 24: 1147-1156, 2013.

2. Printz C: New findings in colon cancer incidence, screening. Cancer 120: 2224-2225, 2014

3. Wang X, Yan D, Teng M, Fan J, Zhou C, Li D, Qiu G, Sun X, Li T, Xing T, et al: Reduced expression of PER3 is associated with incidence and development of colon cancer. Ann Surg Oncol 19: 3081-3088, 2012.

4. Uzgare RP, Sheets TP and Johnston DS: Evaluation of melphalan, oxaliplatin, and paclitaxel in colon, liver, and gastric cancer cell lines in a short-term exposure model of chemosaturation therapy by percutaneous hepatic perfusion. Anticancer Res 33: 1989-2000, 2013.

5. Leone F, Artale S, Marino D, Cagnazzo C, Cascinu S, Pinto C Fornarini G, Tampellini M, Di Fabio F, Sartore-Bianchi A, et al: Panitumumab in combination with infusional oxaliplatin and oral capecitabine for conversion therapy in patients with colon cancer and advanced liver metastases. The MetaPan study. Cancer 119: 3429-3435, 2013

6. Mettu NB, Hurwitz H and Hsu DS: Use of molecular biomarkers to inform adjuvant therapy for colon cancer. Oncology (Williston Park) 27: 746-754, 2013.

7. Kannen V, Garcia SB, Stopper H and Waaga-Gasser AM: Glucagon-like peptide 2 in colon carcinogenesis: Possible target for anti-cancer therapy? Pharmacol Ther 139: 87-94, 2013.

8. Anderson E, Devenney PS, Hill RE and Lettice LA: Mapping the Shh long-range regulatory domain. Development 141: 3934-3943, 2014.

9. Kwong L, Bijlsma MF and Roelink H: Shh-mediated degradation of Hhip allows cell autonomous and non-cell autonomous Shh signalling. Nat Commun 5: 4849, 2014.

10. Filbin MG, Dabral SK, Pazyra-Murphy MF, Ramkissoon S, Kung AL, Pak E, Chung J, Theisen MA, Sun Y, Franchetti Y, et al: Coordinate activation of Shh and PI3K signaling in PTEN-deficient glioblastoma: New therapeutic opportunities. Nat Med 19: 1518-1523, 2013.

11. Song L, Liu Y, Yu Y, Duan X, Qi S and Liu Y: Shh signaling guides spatial pathfinding of raphespinal tract axons by multidirectional repulsion. Cell Res 22: 697-716, 2012.

12. Morton JP and Lewis BC: Shh signaling and pancreatic cancer: Implications for therapy? Cell Cycle 6: 1553-1557, 2007.

13. Vorechovský I, Benediktsson $\mathrm{KP}$ and Toftgård R: The patched/hedgehog/smoothened signalling pathway in human breast cancer: No evidence for H133Y SHH, PTCH and SMO mutations. Eur J Cancer 35: 711-713, 1999.

14. Du P, Ye L, Li H, Yang Y and Jiang WG: The tumour suppressive role of metastasis suppressor-1, MTSS1, in human kidney cancer, a possible connection with the SHH pathway. J Exp Ther Oncol 10: 91-99, 2012.

15. Li X, Wang Z, Ma Q, Xu Q, Liu H, Duan W, Lei J, Ma J, Wang $\mathrm{X}, \mathrm{Lv} \mathrm{S}$, et al: Sonic hedgehog paracrine signaling activates stromal cells to promote perineural invasion in pancreatic cancer. Clin Cancer Res 20: 4326-4338, 2014.

16. Xu X, Su B, Xie C, Wei S, Zhou Y, Liu H, Dai W, Cheng P, Wang F, Xu X and Guo C: Sonic hedgehog-Glil signaling pathway regulates the epithelial mesenchymal transition (EMT) by mediating a new target gene, S100A4, in pancreatic cancer cells. PLoS One 9: e96441, 2014.
17. Chen JS, Huang XH, Wang Q, Huang JQ, Zhang LJ, Chen XL, Lei J and Cheng ZX: Sonic hedgehog signaling pathway induces cell migration and invasion through focal adhesion kinase/AKT signaling-mediated activation of matrix metalloproteinase (MMP)-2 and MMP-9 in liver cancer. Carcinogenesis 34: 10-19, 2013.

18. Omenetti A and Diehl AM: The adventures of sonic hedgehog in development and repair. II. Sonic hedgehog and liver development, inflammation and cancer. Am J Physiol Gastrointest Liver Physiol 294: G595-G598, 2008.

19. Yoshikawa K, Shimada M, Miyamoto H, Higashijima J, Miyatani T, Nishioka M, Kurita N, Iwata T and Uehara H: Sonic hedgehog relates to colorectal carcinogenesis. J Gastroenterol 44: 1113-1117, 2009.

20. Mazumdar T, DeVecchio J, Agyeman A, Shi T and Houghton JA: The GLI genes as the molecular switch in disrupting Hedgehog signaling in colon cancer. Oncotarget 2: 638-645, 2011.

21. Pricl S, Cortelazzi B, Dal Col V, Marson D, Laurini E, Fermeglia M, Licitra L, Pilotti S, Bossi P and Perrone F: Smoothened (SMO) receptor mutations dictate resistance to vismodegib in basal cell carcinoma. Mol Oncol 9: 389-397, 2015

22. O'Kane GM, Lyons T, McDonald I, Mulligan N, Moloney FJ, Murray D and Kelly CM: Vismodegib in the treatment of advanced BCC. Ir Med J 107: 215-216, 2014

23. Petry FR, Nicholls SB, Hébert SS and Planel E: A simple method to avoid nonspecific signal when using monoclonal anti-tau antibodies in western blotting of mouse brain proteins. Methods Mol Biol 1523: 263-272, 2017.

24. Livak KJ and Schmittgen TD: Analysis of relative gene expression data using real-time quantitative PCR and the 2(-Delta Delta C(T)) Method. Methods 25: 402-408, 2001.

25. Hockenbery DM, Zutter M, Hickey W, Nahm M and Korsmeyer SJ: BCL2 protein is topographically restricted in tissues characterized by apoptotic cell death. Proc Natl Acad Sci USA 88: 6961-6965, 1991.

26. Huang EH, Hynes MJ, Zhang T, Ginestier C, Dontu G, Appelman H, Fields JZ, Wicha MS and Boman BM: Aldehyde dehydrogenase 1 is a marker for normal and malignant human colonic stem cells (SC) and tracks SC overpopulation during colon tumorigenesis. Cancer Res 69: 3382-3389, 2009.

27. Lee EY, Ji H, Ouyang Z, Zhou B, Ma W, Vokes SA, McMahon AP, Wong WH and Scott MP: Hedgehog pathway-regulated gene networks in cerebellum development and tumorigenesis. Proc Natl Acad Sci USA 107: 9736-9741, 2010.

28. Chinnaiya K, Tickle C and Towers M: Sonic hedgehog-expressing cells in the developing limb measure time by an intrinsic cell cycle clock. Nat Commun 5: 4230, 2014

29. Tickle $\mathrm{C}$ and Barker $\mathrm{H}$ : The sonic hedgehog gradient in the developing limb. Wiley Interdiscip Rev Dev Biol 2: 275-290, 2013.

30. Wang B, Harrison W, Overbeek PA and Zheng H: Transposon mutagenesis with coat color genotyping identifies an essential role for Skor2 in sonic hedgehog signaling and cerebellum development. Development 138: 4487-4497, 2011.

31. Dessinioti C, Plaka M and Stratigos AJ: Vismodegib for the treatment of basal cell carcinoma: Results and implications of the ERIVANCE BCC trial. Future Oncol 10: 927-936, 2014.

32. Berrada N, Lkhoyali S, Mrabti H and Errihani H: Vismodegib: The proof of concept in Basal cell carcinoma. Clin Med Insights Oncol 8: 77-80, 2014

33. Lyons TG, O'Kane GM and Kelly CM: Efficacy and safety of vismodegib: A new therapeutic agent in the treatment of basal cell carcinoma. Expert Opin Drug Saf 13: 1125-1132, 2014.

34. Yoo YA, Kang MH, Lee HJ, Kim BH, Park JK, Kim HK, Kim JS and Oh SC: Sonic hedgehog pathway promotes metastasis and lymphangiogenesis via activation of Akt, EMT, and MMP-9 pathway in gastric cancer. Cancer Res 71: 7061-7070, 2011.

35. Fan HX, Wang S, Zhao H, Liu N, Chen D, Sun M and Zheng JH: Sonic hedgehog signaling may promote invasion and metastasis of oral squamous cell carcinoma by activating MMP-9 and E-cadherin expression. Med Oncol 31: 41, 2014.

36. Das S, Samant RS and Shevde LA: The hedgehog pathway conditions the bone microenvironment for osteolytic metastasis of breast cancer. Int J Breast Cancer 2012: 298623, 2012.

37. Varnat F, Duquet A, Malerba M, Zbinden M, Mas C, Gervaz P and Ruiz i Altaba A: Human colon cancer epithelial cells harbour active HEDGEHOG-GLI signalling that is essential for tumour growth, recurrence, metastasis and stem cell survival and expansion. EMBO Mol Med 1: 338-351, 2009. 